\title{
Domain General Processes for Interactive Touch
}

Tao Buck ${ }^{1}$, Courtney DiCocco ${ }^{1}$, Jennifer L. Cuzzocreo ${ }^{1,}$ J. Adam Noah ${ }^{1}$, Xian Zhang1, Joy Hirsch*1,2,3,4,5,6

${ }^{1}$ Department of Psychiatry, Yale School of Medicine, New Haven, CT, USA

${ }^{2}$ Department of Comparative Medicine, Yale School of Medicine, New Haven, CT, USA

${ }^{3}$ Department of Neuroscience, Yale School of Medicine, New Haven, CT, USA

${ }^{4}$ Interdepartmental Neuroscience Program, Yale School of Medicine, New Haven, CT, USA

${ }^{5}$ Haskins Laboratories, New Haven, CT, USA

${ }^{6}$ Department of Medical Physics and Biomedical Engineering, University College London, London, UK

*Correspondence:

Joy Hirsch, PhD

joy.hirsch@yale.edu

Keywords: interactive touch, hyperscanning, somatosensory social system, social touch, functional near infrared spectroscopy (fNIRS) 
bioRxiv preprint doi: https://doi.org/10.1101/2021.09.23.461532; this version posted September 24, 2021. The copyright holder for this preprint (which was not certified by peer review) is the author/funder. All rights reserved. No reuse allowed without permission.

Neural Processing of Interactive Touch

\begin{abstract}
The nexus model of social processing proposes that the right temporal parietal junction (rTPJ) serves as a neural hub for cognitive social functions. We test the hypothesis that the rTPJ is a domain general region including somatosensory social functions. Neuroimaging findings and cross-brain coherence for right- and left-hand handclasps with real vs. simulated hands were consistent with the domain general model.
\end{abstract}


Neural Processing of Interactive Touch

\section{Introduction}

Touch plays a fundamental role in natural social and interactive communication. For example, the handshake is a social act wherein two individuals make contact to greet one another or to conclude a social interaction (Hall \& Spencer Hall, 1983). It is commonly used as a proxy for contract agreement that conveys trust (Dolcos et al., 2012). Importantly, a handshake creates an interactive union between two individuals and represents a form of nonverbal social communication (Hall, 2003). Similar to communicating through eye contact or a verbal dialogue, the handshake is an action that has an intention and involves interpersonal feedback. Understanding the neural mechanisms of natural interactions has been challenging due, in part, to technological limitations associated with observations of live interplay between dyads. However, these limitations have been largely resolved by advances in functional near infrared spectroscopy (fNIRS), a non-invasive head mounted spectral absorbance approach, that measures task-related changes in blood oxygen levels in both oxyhemoglobin and deoxyhemoglobin through optical sensors (Jöbsis, 1977; Strangman et al., 2002; Villringer \& Chance, 1997). This method allows the recording of hemodynamic signals from two participants simultaneously and has enabled an increased focus on live and natural interactions (Cui et al., 2012; Hasson \& Frith, 2016; Hasson et al., 2012; Hirsch et al., 2018; Redcay et al., 2012; Schilbach et al., 2013).

Social touch, such as a handshake or a hand clasp, conveys both somatosensory and interpersonal social information. Although somatosensory pathways are well known, the neural systems associated with social touch information are less well characterized. We distinguish between two alternative models for somatosensory social information: in one case this social information is processed within the contralateral primary and association systems; and in the alternative case the social information is processed separately from the somatosensory information. The Nexus model of social behavior (Carter \& Huettel, 2013) proposes that the right temporal parietal junction (rTPJ) integrates multiple streams of cognitive and social information in order to provide a social context, including theory-of-mind functions. Previous studies have found that the right TPJ is also sensitive to live interactions, including eye contact, consistent with social interactive behavior (Kelley et al., 2021; Noah et al., 2020). These visual processes, also with a social meaning, suggest that the rTPJ may be a domain general region for social and interactive processes.

To test this hypothesis for social touch, we use the sensorimotor system and the social task of hand clasping. We employ a hand clasp with a live partner and compare responses with a hand clasp with a simulated hand in order to distinguish a "social" condition from a "non-social" condition. The design of this study takes advantage of the anatomically crossed sensory and motor pathways where expected responses for touch include contralateral regions in sensorimotor hand areas. The hypothesis of a domain general function for rTPJ would be supported if the right TPJ is also be active during social hand-clasping (either right or left hand) and is greater for real hands rather than for simulated hands. In this investigation, fNIRS was used to concurrently record hemodynamic signals from dyads during left and right hand-clasping intended to represent the initial connection made in a common handshake.

\section{Methods}

\subsection{Participants}

Thirty healthy adults (15 pairs; 19 females; mean age: $28.8 \pm 12.2$ years; 29 right-handed (Oldfield, 1971)), took part in this study. There were 6 female-female, 2 male-male, and 7 female-male pairs. No known neurologic or psychiatric conditions were reported. Written informed consent was provided by all participants in accordance with the guidelines approved by the Yale University Human Investigation Committee (HIC \#1501015178), and each person was compensated for 
participating. Participants who were previously unacquainted were recruited and paired prior to the experiment. All participants had performed a finger-thumb tapping screening task at an earlier date to confirm the presence of recordable hemodynamic signals. See Table S1 for individual participant and group summary demographics.

A.

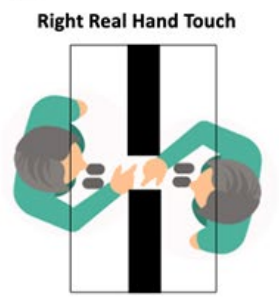

Left Plastic Hand Touch

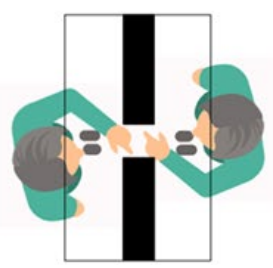

B.

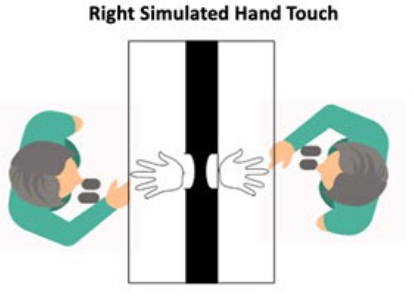

Left Simulated Hand Touch

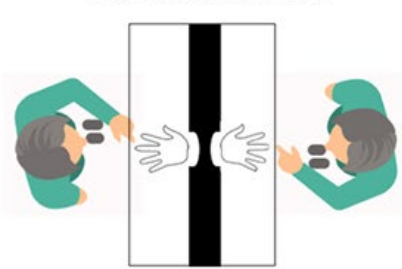

C.

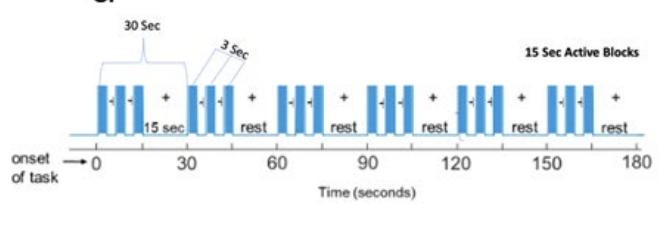

D.

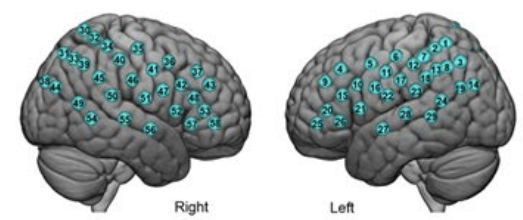

Figure 1. Experimental conditions, time series, and channel layout. A. Real Hand condition. Partners faced each other at a distance of $140 \mathrm{~cm}$ with an occlusion between them that prohibited them from seeing one another but allowed hand contact. Participants were asked to grasp their partner's left and right hand. B. Simulated Hand condition. Participants were asked to grasp a simulated plastic hand arranged on the table in front of them in the same location as their partner's hand during the real hand condition. C. Time course of the experimental paradigm. The entire duration of every run was three minutes, and each run was repeated twice for the Real Hand and Simulated Hand (right and left hands), for a total of eight runs. Each run included six alternating 15second task and rest periods. In the task period (blue bars), participants grasped their partner's hand or a simulated hand in three-second periods alternating with three-second periods of gaze fixation on a crosshair located on the occluder at eye level. During the 15-second rest period, participants looked at a crosshair and rested the hands on the table. D. Channel layout. Right and left hemispheres of a single rendered brain illustrate median locations (blue dots) for 58 channels per participant. Montreal Neurological Institute (MNI) coordinates were determined for each channel (numbers on blue dots) by digitizing emitter and detector locations in relation to anterior, posterior, dorsal, and lateral fiduciary markers based on the standard 10-20 system.

\subsection{Paradigm and Procedures}

Dyads participated in four tasks (Figure 1A \& 1B) during which they clasped right or left hands, as if to initiate a handshake, with either a partner or a simulated hand (i.e., they clasped a real hand or simulated hand with their right hand or left hand). Partners were seated at a table approximately 140 $\mathrm{cm}$ from one another. An occluder panel on the table between participants prevented them from seeing each other but allowed them to clasp the other person's hand in the Real Hand conditions. Simulated hands were arranged in the middle of the table where the partners' hands met. The order of tasks was arranged randomly, and each condition was repeated twice before proceeding to the next. The timeseries of each condition (Figure 1C) is similar to that used in prior studies (Hirsch et al., 2018; Hirsch et al., 2017; Noah et al., 2020; Piva et al., 2017). All runs consisted of 30-second blocks with the active task period alternating with a 15 -second rest/baseline period for a total of 180 seconds per condition. An initial auditory cue instructed participants to grasp the simulated hand in front of them or grasp the other participant's hand. Every three seconds, an auditory tone prompted the dyad to hold 
Neural Processing of Interactive Touch

or let go of the hand. Participants focused their gaze on a crosshair located on the occluder during the $3 \mathrm{~s}$ in which the participants were not clasping hands (real or simulated) and during the $15 \mathrm{~s}$ rest/baseline period.

\subsection{Signal Acquisition and Channel Localization}

Signal acquisition, optode localization, and signal processing, including global mean removal, were similar to methods described previously (Dravida et al., 2018; Dravida et al., 2020; Hirsch et al., 2018; Hirsch et al., 2017; Kelley et al., 2021; Noah et al., 2017; Noah et al., 2015; Noah et al., 2020; Piva et al., 2017; Zhang et al., 2017; Zhang et al., 2016) and are consistent with recommended standards of practice (Yücel et al., 2021). Hemodynamic signals were acquired using an 80-fiber multichannel, continuous-wave fNIRS system (LABNIRS, Shimadzu Corp., Kyoto, Japan) that emits and detects three wavelengths of light $(780 \mathrm{~nm}, 805 \mathrm{~nm}, 830 \mathrm{~nm})$. Each participant was fitted with an optode cap with predefined channel distances between holders. Three sizes of caps were used based on the circumference of the heads of subjects. Large caps had a $60 \mathrm{~cm}$ circumference. Medium caps were $56.5 \mathrm{~cm}$ and small caps were $54.5 \mathrm{~cm}$. Optode distances of $3 \mathrm{~cm}$ were designed for the $60 \mathrm{~cm}$ cap layout but were scaled equally to smaller caps. To remove hair from each channel opening, a lighted fiber-optic probe (Daiso, Hiroshima, Japan) was used prior to optode placement. Forty emitter and detector optode pairs were organized to record from 58 channels per participant (Figure 1D). Using a modified Beer-Lambert equation (Hazeki \& Tamura, 1988; Hoshi, 2003; Matcher et al., 1995), the absorbed light measured by the detectors was translated into concentrations of oxyhemoglobin $(\mathrm{OxyHb})$ and deoxyhemoglobin (deOxyHb). Signals were acquired at $30 \mathrm{~Hz}$. Resistance was evaluated and adjusted for each channel before starting the experiment to ensure that the data obtained would have an acceptable signal-to-noise ratio. Anatomical locations of optodes in relation to standard head landmarks were determined for each participant using a Patriot 3D Digitizer (Polhemus, Colchester, VT) (Eggebrecht et al., 2014; Eggebrecht et al., 2012; Ferradal et al., 2014; Matcher et al., 1995; Okamoto \& Dan, 2005; Singh et al., 2005). Montreal Neurological Institute (MNI) coordinates (Mazziotta et al., 2001) for each channel were obtained using NIRS-SPM software (Ye et al., 2009). Anatomical regions corresponding to channel locations were identified with WFU Pickatlas (Maldjian et al., 2004; Maldjian et al., 2003).

\subsection{Signal Processing and statistical analysis}

Channels containing excessive noise were determined and rejected by calculating any channel with raw data having a root mean square 10 times larger than the sum of all the channels root mean square. Data were then converted to concentrations of oxyhemoglobin (OxyHb) and deoxyhemoglobin (deOxyHb) chromophore concentrations using the Beer-Lambert Law (Hazeki \& Tamura, 1988; Hoshi, 2003; Matcher et al., 1995). The wavelet detrending function available in NIRS-SPM software was applied to remove baseline drift (Ye et al., 2009). A principal component analysis (PCA) and global-mean filter (Zhang et al., 2017; Zhang et al., 2016) was applied to remove global components due to physiologic factors including blood pressure and other non-neural components (Tachtsidis \& Scholkmann, 2016) prior to a general linear model (GLM) analysis. No additional pre-processing steps were performed.

General linear modeling analysis was performed on the hemoglobin difference signal $\left([\mathrm{Hbdiff}]=\left[\mathrm{HbO}_{2}\right]-[\mathrm{HHb}]\right)($ Tachtsidis et al., 2009), referred to here as the combined signal, where the signs of both the $\mathrm{OxyHb}\left(\mathrm{HbO}_{2}\right)$ and $\mathrm{deOxyHb}(\mathrm{HHb})$ signals are consistent with true brain activity. Event periods (Figure 1C) were convolved with the canonical hemodynamic response function supplied by SPM8 (Penny et al., 2011) to create modeled time courses for obtaining beta values using the GLM approach. The differences between the individual beta values across conditions were interpolated into an MNI brain template for second level analysis, yielding contrast results. 
Significance was determined using one-tailed t-tests. Findings were corrected using False Discovery Rate (FDR) at $\mathrm{p} \leq 0.05$ within MATLAB.

A finger-thumb tapping localizer task was performed to confirm the expected hand/finger localizations (Dravida, et al, 2018). Fifteen sec epochs of finger-thumb tapping alternated with 15 secs of rest for a total of six cycles and $180 \mathrm{sec}$. Two runs were acquired for each hand. The order of hands was counterbalanced. Observation of the expected contralateral pre-motor and supplementary motor localizations for right- and left-hand activity compared to rest is shown in Figure 2 and Table 1A and 1B.

\section{A. Left Hand Localizer}

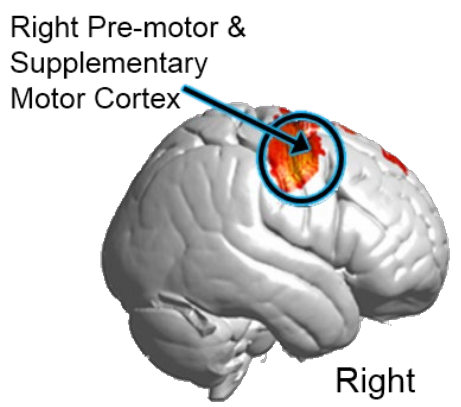

\section{B. Right Hand Localizer}

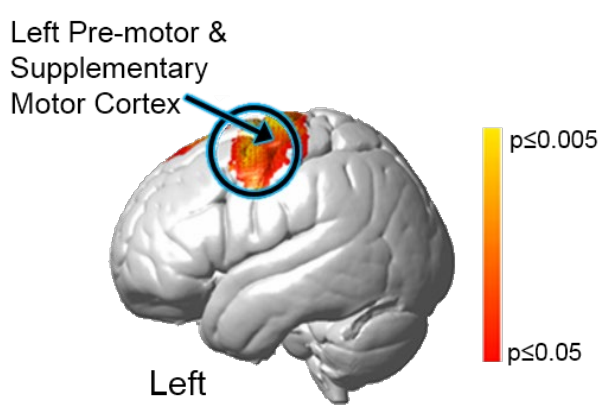

Figure 2. A. Right Hand Localizer task: contrast comparison [Real Hand $>$ Rest] based on the combined $\mathrm{OxyHb}$ and deOxyHb signals $(\mathrm{p} \leq 0.05)$. Activity was observed in left pre-motor and supplementary motor cortex (See Table 1A). B. Left Hand Localizer task: contrast comparison [Real Hand $>$ Rest] based on the combined OxyHb and deOxyHb signals $(\mathrm{p} \leq 0.05)$. Activity was observed in right and left pre-motor and supplementary motor cortex (See Table 1B).

Cross-brain synchrony (coherence) was analysed for the real hand condition versus the simulated hand using wavelet analysis (Torrence \& Compo, 1998) from the MATLAB 2018A Wavelet Toolbox. Signals were decomposed into a range of temporal frequencies. Coherence was calculated for each frequency component (y-axis) as a function of component wavelength (period, $\mathrm{x}$ axis) (Hirsch et al., 2018; Hirsch et al., 2021; Noah et al., 2020).

\section{$3 \quad$ Results}

\subsection{GLM Analysis}

[Real Right and Left Hand > Simulated Right and Left Hand]
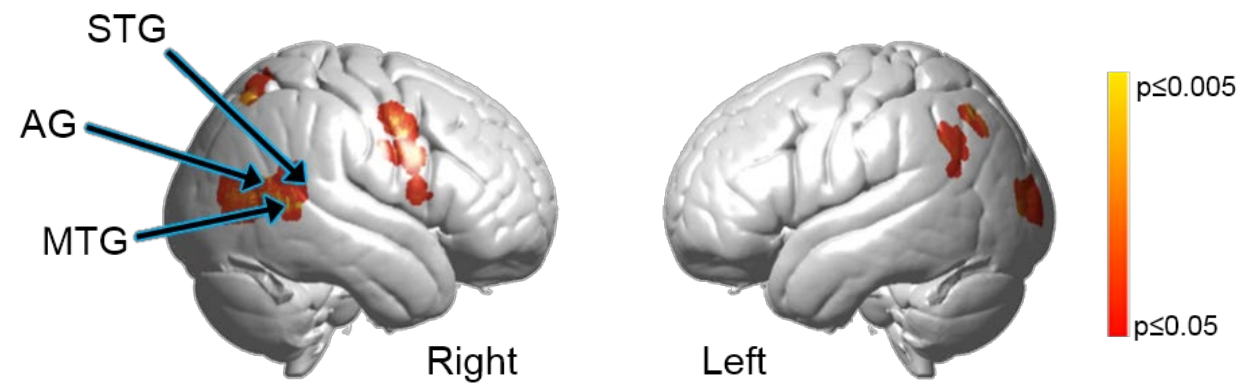
Neural Processing of Interactive Touch

Figure 3. Contrast comparison of all [Real Right and Left Hand > Simulated Right and Left Hand] activity, combining both right and left hands, based on the combined $\mathrm{OxyHb}$ and deOxyHb signals $(\mathrm{p} \leq 0.05)$. Activity was observed in right hemisphere superior temporal gyrus (STG); middle temporal gyrus (MTG); and angular gyrus (AG), components of the rTPJ. See Table 2.

A voxel-wise general linear model (GLM) contrasting the tasks involving the real hand versus the simulated hand combining left and right hands shows activation in the temporoparietal junction in the right hemisphere (Figure 3 and Table 2). These areas include the right superior temporal gyrus (STG), middle temporal gyrus (MTG), and angular gyrus (AG) consistent with social and interactive processing in the rTPJ confirming the domain general hypothesis

\subsection{Cross-Brain Neural Coherence}

In addition to a cognitive social context, such as the theory-of-mind conventionally investigated with single participant paradigms (Carter \& Huettel, 2013), it has been shown that the rTPJ also codes live interactions such as face-to-face processes (Kelley et al., 2021; Noah et al., 2020). Domain general processes for live interactions predicts cross-brain coherence between TPJ regions for social touch (Hasson et al., 2012). In accordance with this prediction, coherence was greater during the interactive real-hand tasks than simulated hand tasks (Figure 4).

Temporal oscillations of hemodynamic signals decomposed into wavelet components (x-axis) are shown against the correlation between the signals of partners (y-axis) acquired during the joint tasks of grasping the real and the simulated hands, red and blue respectively (methods described previously (Hirsch et al., 2018; Hirsch et al., 2017; Noah et al., 2020). Cross-brain coherence between the superior temporal and somatosensory gyri was greater in the real hand task (red) in comparison to the simulated hand task (blue). Interestingly, this was true only for the case of real partners (Fig 4A), and not for shuffled partners (Fig 4B), (i.e., randomly paired with every other subject except the original partner) where no difference in coherence was found. This comparison is consistent with the interpretation that cross-brain signal coherence was due to the specific interactions between the participants performing the real hand clasp.

Real Partners

Shuffled Partners

Superior Temporal Gyrus to Somatosensory Cortex
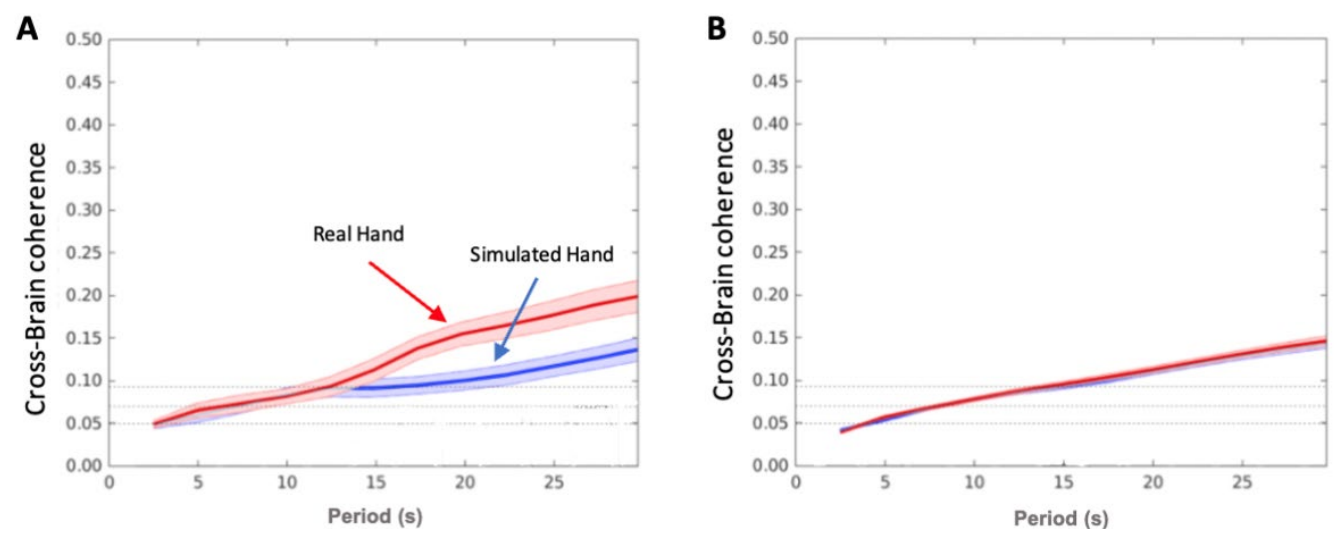

Figure 4. Brain-to-brain coherence between dyads (y-axis) is plotted against the temporal period (wavelength) (x-axis) for the real hand condition (right and left) (red) and the simulated hand condition (right and left) (blue) (surrounding colors \pm 1 SEM). A. depicts coherence between real 
Neural Processing of Interactive Touch

partners whereas B. shows coherence between shuffled partners. Cross-brain coherence is highest in the real hand conditions between superior temporal gyrus to somatosensory cortex.

\section{Conclusion}

These findings advance our understanding of the neural systems that underlie social cognition and social touch consistent with a domain general function for the rTPJ. Although the rTPJ has been shown to play a role in social cognition, such as theory of mind (Carter \& Huettel, 2013), as well as live face-to-face gaze (Kelley et al., 2021; Noah et al., 2020), this is the first documentation of a role in processing social touch and provides evidence in support of rTPJ as a domain general region for social and interactive functions. Further, findings of increased cross-brain coherence of rTPJ signals between partners during real hand clasping relative to simulated hand clasping are consistent with neural coupling between the interactive systems. 
Neural Processing of Interactive Touch

\section{$5 \quad$ Conflict of Interest}

The authors declare that the research was conducted in the absence of any commercial or financial relationships that could be construed as a potential conflict of interest.

\section{Author Contributions}

J.H. designed and supervised this experiment. C.D., A.N, X.Z., J.L.C., and T.B. performed the experiment and analyzed the data. T.B. and J.H. wrote the manuscript.

\section{$7 \quad$ Funding and Acknowledgements}

This research was partially supported by the National Institute of Mental Health of the National Institutes of Health under award numbers 1R01MH111629 (PIs JH and JM); R01MH107513 (PI JH); 1R01MH119430 (PI JH). The content is solely the responsibility of the authors and does not necessarily represent the official views of the National Institutes of Health. All data reported in this paper are available upon request from the corresponding author.

\section{Data Availability Statement}

The datasets analyzed for this study will be made available upon request at fmri.org 
Neural Processing of Interactive Touch

\section{References}

Carter, R. M., \& Huettel, S. A. (2013). A nexus model of the temporal-parietal junction. Trends in cognitive sciences, 17(7), 328-336. doi: 10.1016/j.tics.2013.05.007

Cui, X., Bryant, D. M., \& Reiss, A. L. (2012). NIRS-based hyperscanning reveals increased interpersonal coherence in superior frontal cortex during cooperation. Neuroimage, 59(3), 2430-2437. doi: 10.1016/j.neuroimage.2011.09.003

Dolcos, S., Sung, K., Argo, J. J., Flor-Henry, S., \& Dolcos, F. (2012). The power of a handshake: neural correlates of evaluative judgments in observed social interactions. Journal of Cognitive Neuroscience, 24(12), 2292-2305.

Dravida, S., Noah, J. A., Zhang, X., \& Hirsch, J. (2018). Comparison of oxyhemoglobin and deoxyhemoglobin signal reliability with and without global mean removal for digit manipulation motor tasks. Neurophotonics, 5(1), 011006. doi: 10.1117/1.NPh.5.1.011006

Dravida, S., Noah, J. A., Zhang, X., \& Hirsch, J. (2020). Joint attention during live person-to-person contact activates rTPJ, including a sub-component associated with spontaneous eye-to-eye contact. Frontiers in human neuroscience, 14. doi: 10.3389/fnhum.2020.00201

Eggebrecht, A. T., Ferradal, S. L., Robichaux-Viehoever, A., Hassanpour, M. S., Dehghani, H., Snyder, A. Z., Hershey, T., \& Culver, J. P. (2014). Mapping distributed brain function and networks with diffuse optical tomography. Nature Photonics, 8(6), 448.

Eggebrecht, A. T., White, B. R., Ferradal, S. L., Chen, C., Zhan, Y., Snyder, A. Z., Dehghani, H., \& Culver, J. P. (2012). A quantitative spatial comparison of high-density diffuse optical tomography and fMRI cortical mapping. Neuroimage, 61(4), 1120-1128.

Ferradal, S. L., Eggebrecht, A. T., Hassanpour, M., Snyder, A. Z., \& Culver, J. P. (2014). Atlasbased head modeling and spatial normalization for high-density diffuse optical tomography: In vivo validation against fMRI. Neuroimage, $85,117-126$.

Hall, P. M. (2003). Interactionism, social organization, and social processes: Looking back and moving ahead. Symbolic Interaction, 26(1), 33-55.

Hall, P. M., \& Spencer Hall, D. A. (1983). The handshake as interaction.

Hasson, U., \& Frith, C. D. (2016). Mirroring and beyond: coupled dynamics as a generalized framework for modelling social interactions. Philosophical Transactions of the Royal Society B: Biological Sciences, 371(1693), 20150366.

Hasson, U., Ghazanfar, A. A., Galantucci, B., Garrod, S., \& Keysers, C. (2012). Brain-to-brain coupling: a mechanism for creating and sharing a social world. Trends in cognitive sciences, 16(2), 114-121.

Hazeki, O., \& Tamura, M. (1988). Quantitative analysis of hemoglobin oxygenation state of rat brain in situ by near-infrared spectrophotometry. Journal of Applied Physiology, 64(2), 796802. doi: 10.1152/jappl.1988.64.2.796

Hirsch, J., Noah, J. A., Zhang, X., Dravida, S., \& Ono, Y. (2018). A Cross-Brain Neural Mechanism for Human-to-Human Verbal Communication. Social Cognitive and Affective Neuroscience. doi: 10.1093/scan/nsy070

Hirsch, J., Tiede, M., Zhang, X., Noah, J. A., Salama-Manteau, A., \& Biriotti, M. (2021). Interpersonal Agreement and Disagreement During Face-to-Face Dialogue: An fNIRS Investigation. Frontiers in human neuroscience, 14, 601. doi: 10.3389/fnhum.2020.606397

Hirsch, J., Zhang, X., Noah, J. A., \& Ono, Y. (2017). Frontal temporal and parietal systems synchronize within and across brains during live eye-to-eye contact. Neuroimage, 157, 314330. doi: 10.1016/j.neuroimage.2017.06.018

Hoshi, Y. (2003). Functional near-infrared optical imaging: Utility and limitations in human brain mapping. Psychophysiology, 40(4), 511-520. doi: Doi 10.1111/1469-8986.00053 
Neural Processing of Interactive Touch

Jöbsis, F. F. (1977). Noninvasive, infrared monitoring of cerebral and myocardial oxygen sufficiency and circulatory parameters. Science, 198(4323), 1264-1267. doi:

10.1126/science.929199

Kelley, M., Noah, J. A., Zhang, X., Scassellati, B., \& Hirsch, J. (2021). Comparison of human social brain activity during eye-contact with another human and a humanoid robot. Frontiers in Robotics and AI, 7, 209.

Maldjian, J. A., Laurienti, P. J., \& Burdette, J. H. (2004). Precentral gyrus discrepancy in electronic versions of the Talairach atlas. Neuroimage, 21(1), 450-455.

Maldjian, J. A., Laurienti, P. J., Kraft, R. A., \& Burdette, J. H. (2003). An automated method for neuroanatomic and cytoarchitectonic atlas-based interrogation of fMRI data sets. Neuroimage, 19(3), 1233-1239.

Matcher, S. J., Elwell, C. E., Cooper, C. E., Cope, M., \& Delpy, D. T. (1995). Performance comparison of several published tissue near-infrared spectroscopy algorithms. Analytical Biochemistry, 227(1), 54-68. doi: 10.1006/abio.1995.1252

Mazziotta, J., Toga, A., Evans, A., Fox, P., Lancaster, J., Zilles, K., Woods, R., Paus, T., Simpson, G., \& Pike, B. (2001). A probabilistic atlas and reference system for the human brain: International Consortium for Brain Mapping (ICBM). Philosophical Transactions of the Royal Society B: Biological Sciences, 356(1412), 1293-1322.

Noah, J. A., Dravida, S., Zhang, X., Yahil, S., \& Hirsch, J. (2017). Neural correlates of conflict between gestures and words: A domain-specific role for a temporal-parietal complex. PLoS ONE, 12(3), e0173525. doi: 10.1371/journal.pone.0173525

Noah, J. A., Ono, Y., Nomoto, Y., Shimada, S., Tachibana, A., Zhang, X., Bronner, S., \& Hirsch, J. (2015). fMRI validation of fNIRS measurements during a naturalistic task. Journal of Visualized Experiments: JoVE(100).

Noah, J. A., Zhang, X., Dravida, S., Ono, Y., Naples, A., McPartland, J. C., \& Hirsch, J. (2020). Real-time eye-to-eye contact is associated with cross-brain neural coupling in angular gyrus. Frontiers in Human Neuroscience, 14, 19. doi: 10.3389/fnhum.2020.00019

Okamoto, M., \& Dan, I. (2005). Automated cortical projection of head-surface locations for transcranial functional brain mapping. Neuroimage, 26(1), 18-28.

Oldfield, R. C. (1971). The assessment and analysis of handedness: The Edinburgh inventory. Neuropsychologia, 9(1), 97-113.

Penny, W. D., Friston, K. J., Ashburner, J. T., Kiebel, S. J., \& Nichols, T. E. (2011). Statistical parametric mapping: the analysis of functional brain images: Elsevier.

Piva, M., Zhang, X., Noah, J. A., Chang, S. W., \& Hirsch, J. (2017). Distributed neural activity patterns during human-to-human competition. Frontiers in human neuroscience, 11, 571.

Redcay, E., Kleiner, M., \& Saxe, R. (2012). Look at this: The neural correlates of initiating and responding to bids for joint attention. Frontiers in human neuroscience, 6, 169.

Schilbach, L., Timmermans, B., Reddy, V., Costall, A., Bente, G., Schlicht, T., \& Vogeley, K. (2013). Toward a second-person neuroscience. Behavioral and Brain Sciences, 36(04), 393414. doi: $10.1017 / \mathrm{S} 0140525 \mathrm{X} 12000660$

Singh, A. K., Okamoto, M., Dan, H., Jurcak, V., \& Dan, I. (2005). Spatial registration of multichannel multi-subject fNIRS data to MNI space without MRI. Neuroimage, 27(4), 842851.

Strangman, G., Culver, J. P., Thompson, J. H., \& Boas, D. A. (2002). A quantitative comparison of simultaneous BOLD fMRI and NIRS recordings during functional brain activation.

Neuroimage, 17(2), 719-731.

Tachtsidis, I., \& Scholkmann, F. (2016). False positives and false negatives in functional nearinfrared spectroscopy: Issues, challenges, and the way forward. Neurophotonics, 3(3), 031405. doi: 10.1117/1.NPh.3.3.031405 
Neural Processing of Interactive Touch

Tachtsidis, I., Tisdall, M. M., Leung, T. S., Pritchard, C., Cooper, C. E., Smith, M., \& Elwell, C. E. (2009). Relationship between brain tissue haemodynamics, oxygenation and metabolism in the healthy human adult brain during hyperoxia and hypercapnea Oxygen Transport to Tissue $X X X$ (pp. 315-320): Springer.

Torrence, C., \& Compo, G. P. (1998). A practical guide to wavelet analysis. Bulletin of the American Meteorological society, 79(1), 61-78.

Villringer, A., \& Chance, B. (1997). Non-invasive optical spectroscopy and imaging of human brain function. Trends in Neurosciences, 20(10), 435-442.

Ye, J. C., Tak, S., Jang, K. E., Jung, J., \& Jang, J. (2009). NIRS-SPM: statistical parametric mapping for near-infrared spectroscopy. Neuroimage, 44(2), 428-447.

Yücel, M. A., Lühmann, A. v., Scholkmann, F., Gervain, J., Dan, I., Ayaz, H., Boas, D., Cooper, R. J., Culver, J., \& Elwell, C. E. (2021). Best practices for fNIRS publications. Neurophotonics, 8(1), 012101.

Zhang, X., Noah, J. A., Dravida, S., \& Hirsch, J. (2017). Signal processing of functional NIRS data acquired during overt speaking. Neurophotonics, 4(4), 041409. doi: 10.1117/1.NPh.4.4.041409

Zhang, X., Noah, J. A., \& Hirsch, J. (2016). Separation of the global and local components in functional near-infrared spectroscopy signals using principal component spatial filtering. Neurophotonics, 3(1), 015004. 
bioRxiv preprint doi: https://doi.org/10.1101/2021.09.23.461532; this version posted September 24, 2021. The copyright holder for this preprint (which was not certified by peer review) is the author/funder. All rights reserved. No reuse allowed without permission.

Neural Processing of Interactive Touch

\section{Tables}

Table 1A. Voxel-wise GLM Contrast comparisons (Oxy+deOxyHb signals), Right Hand Localizer [Real Hand > Rest]

\begin{tabular}{|c|c|c|c|c|c|c|c|c|c|c|c|}
\hline & Contrast & \multicolumn{5}{|c|}{ Peak Voxels } & \multicolumn{3}{|c|}{ Anatomical Regions } & Probability & $\begin{array}{c}\mathrm{n} \text { of } \\
\text { Voxels }\end{array}$ \\
\hline $\begin{array}{c}\text { Left } \\
\text { Hemisphere }\end{array}$ & $\mathrm{p}=0.05$ & -20 & 0 & 68 & 5.13 & 0.001 & 9 & Pre- and Supplementary Motor Cortex & 6 & 1.00 & 2282 \\
\hline
\end{tabular}

${ }^{1}$ Coordinates are based on the MNI system and (-) indicates left hemisphere. ${ }^{2} \mathrm{df}=$ degrees of freedom. ${ }^{3} \mathrm{BA}=$ Brodmann Area.

Table 1B. Voxel-wise GLM Contrast comparison (Oxy+deOxyHb signals), Left Hand Localizer [Real Hand > Rest]

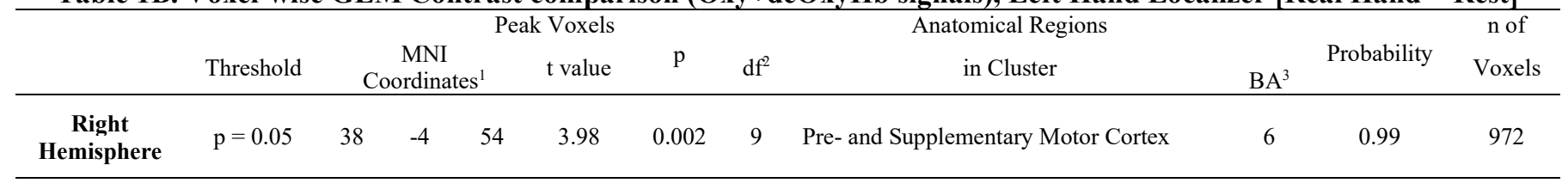

${ }^{1}$ Coordinates are based on the MNI system and (-) indicates left hemisphere. ${ }^{2} \mathrm{df}=$ degrees of freedom. ${ }^{3} \mathrm{BA}=$ Brodmann Area.

Table 2. Voxel-wise GLM Contrast comparisons (Oxy+deOxyHb signals), [Real Hands $>$ Simulated Hands]

\begin{tabular}{|c|c|c|c|c|c|c|c|c|c|c|c|}
\hline \multirow{3}{*}{\begin{tabular}{|c|} 
Contrast \\
[Real Hands >
\end{tabular}} & \multirow{3}{*}{$\begin{array}{c}\text { Contrast } \\
\text { Threshold } \\
p=0.05\end{array}$} & \multicolumn{6}{|c|}{ Peak Voxels } & \multirow{2}{*}{$\begin{array}{l}\text { Anatomical Regions } \\
\text { in Cluster }\end{array}$} & \multirow{2}{*}{\multicolumn{2}{|c|}{$\mathrm{BA}^{3}$ Probability }} & \multirow{2}{*}{$\begin{array}{c}\mathrm{n} \text { of } \\
\text { Voxels }\end{array}$} \\
\hline & & \multicolumn{3}{|c|}{$\begin{array}{c}\text { MNI } \\
\text { Coordinates }\end{array}$} & t value & $\mathrm{p}$ & $\mathrm{df}^{2}$ & & & & \\
\hline & & 68 & -50 & 6 & 3.19 & 0.001 & 29 & Superior Temporal Gyrus & 22 & 0.50 & 390 \\
\hline \multirow[t]{4}{*}{ Simulated Hands] } & & & & & & & & Middle Temporal Gyrus & 21 & 0.39 & \\
\hline & $\mathrm{p}=0.05$ & 50 & -2 & 40 & 2.42 & 0.011 & 29 & Pre- and Supplementary Motor Cortex & 6 & 0.85 & 124 \\
\hline & $\mathrm{p}=0.05$ & 56 & 2 & 26 & 2.16 & 0.020 & 29 & Pre- and Supplementary Motor Cortex & 6 & 0.57 & 87 \\
\hline & & & & & & & & Pars Opercularis & 44 & 0.26 & \\
\hline \multirow[t]{5}{*}{ Right Hemisphere } & & & & & & & & Pars Triangularis & 45 & 0.20 & \\
\hline & & & & & & & & Pre- and Supplementary Motor Cortex & 6 & 0.19 & \\
\hline & $\mathrm{p}=0.05$ & 40 & -64 & 54 & 2.49 & 0.009 & 29 & Somatosensory Association Cortex & 7 & 0.69 & 29 \\
\hline & & & & & & & & Supramarginal Gyrus & 40 & 0.26 & \\
\hline & $\mathrm{p}=0.05$ & -60 & -56 & 34 & 1.99 & 0.028 & 29 & Supramarginal Gyrus & 40 & 0.66 & 24 \\
\hline \multirow{2}{*}{ Left Hemisphere } & & & & & & & & Angular Gyrus & 39 & 0.32 & \\
\hline & & & & & & & & Somatosensory Association Cortex & 7 & 0.27 & \\
\hline
\end{tabular}

${ }^{1}$ Coordinates are based on the MNI system and (-) indicates left hemisphere. ${ }^{2} \mathrm{df}=$ degrees of freedom. ${ }^{3} \mathrm{BA}=$ Brodmann Area. 